\title{
Unusual Sonochemical Assembly between Carbon Allotropes for High Strain-Tolerant Conductive Nanocomposites
}

\author{
Zhaoliang Zheng, ${ }^{\dagger, \#}$ Jidong Jin, ${ }^{\ddagger}$ Jin-Chao Dong, ${ }^{\S}$ Bo Li, ${ }^{\| \odot ~ G u a n g-K u i ~ X u, ~}{ }^{\perp}$ Jian-Feng Li, ${ }^{\S}$ \\ and Dmitry G. Shchukin**0
}

${ }^{\dagger}$ Stephenson Institute for Renewable Energy and Department of Chemistry, University of Liverpool, Liverpool L69 7ZD, United Kingdom

${ }^{\ddagger}$ Department of Chemistry, Chemistry Research Laboratory, University of Oxford, Oxford OX1 3TA, United Kingdom

${ }^{\S}$ MOE Key Laboratory of Spectrochemical Analysis and Instrumentation, State Key Laboratory of Physical Chemistry of Solid Surfaces, College of Chemistry and Chemical Engineering, Xiamen University, Xiamen 361005, China

"Institute of Biomechanics and Medical Engineering, AML, Department of Engineering Mechanics, Tsinghua University, Beijing 100084, China

${ }^{\perp}$ International Center for Applied Mechanics, State Key Laboratory for Strength and Vibration of Mechanical Structures, Xi'an Jiaotong University, Xi'an 710049, China

\section{Supporting Information}

ABSTRACT: Facile methods toward strain-tolerant graphenebased electronic components remain scarce. Although being frequently used to disperse low-dimensional carbonaceous materials, ultrasonication (US) has never been reliable for fabricating stretchable carbonaceous nanocomposite (SCNC). Inspired by the unusual sonochemical assembly between graphene oxide (GO) and carbon nanotube (CNT), we verified the roots-like GO-CNT covalent bonding, rather than just $\pi-\pi$ conjugation, was formed during US. In addition, the shockwaveinduced collision in the binary-component system enables a burst of fragmentation at the early stage, spatially homogeneous

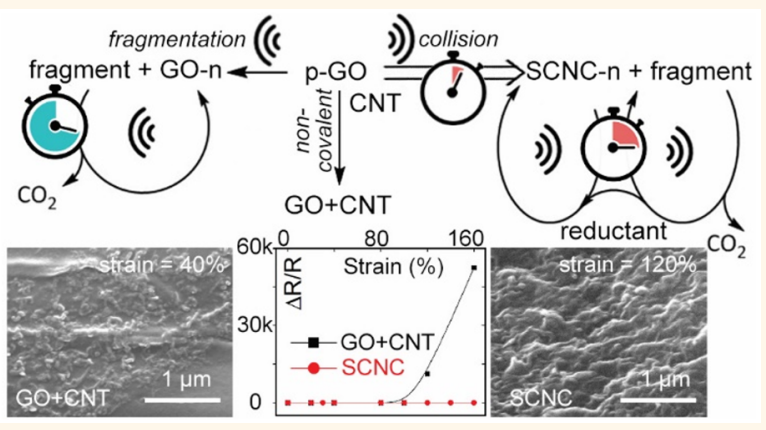
hybridization, and time-dependent restoration of graphitic domains. All of the above are distinct from extensive fragmentation of a conventional single-component system and $\pi-\pi$ conjugative assembly. The optimized SCNC exhibits conductivity comparable to reduced monolayer GO and outperforms $\pi-\pi$ assemblies in retaining electrical conductance at a strain of $160 \%$-among one of the best reported stretchable conductors. Raman analysis and mechanics simulation confirm the dominant role of counterweighing between the intrinsic and external strains on the mechano-response and durability of SCNC. This work suggests the guideline of creating multiple-component sonochemical systems for various functional nanocomposites.

KEYWORDS: sonochemistry, graphene oxide, carbon nanotube, nanocomposites, Raman spectroscopy, stretchable electronics

$\mathrm{T}$ he rapid development of the stretchable electronics inspired the pursuit of strain-tolerant conductive components that retain the integrity of structure and conductivity even under large strains. ${ }^{1,2}$ Two-dimensional (2D) graphene has been reckoned as a highly promising candidate due to its intrinsic tensile strength (130 GPa), flexibility, low resistivity $\left(10^{-6} \Omega \cdot \mathrm{cm}\right)$ and negative Poisson's ratio when the tensile strain exceeds $6 \%{ }^{3,4}$ Disappointingly, the overall conductivity of most reported graphene sheets is retained at less than $5 \%$ strain due to brittle fracture at unavoidable defect or strain-induced inhomogeneity of charge carriers mobility. ${ }^{5-8}$ To enhance the strain tolerance, the monatomic graphene has been geometrically engineered into the mechano-responsive patterns, such as crumple ${ }^{9,10}$ and kirigami. ${ }^{11}$ Meanwhile, the assembly between $2 \mathrm{D}$ graphene and its deformed states (scrolls ${ }^{12,13}$ and crumpled ball ${ }^{14}$ ) or carbon allotropes $^{15}$ provide scalable alternatives. However, the critically high concentration of additives induces the strong dependence of stretchability on the tricky preparative methods and the nature of additives. So far, a facile and easier-handling

Received: August 11, 2019

Accepted: September 18, 2019

Published: September 18, 2019 

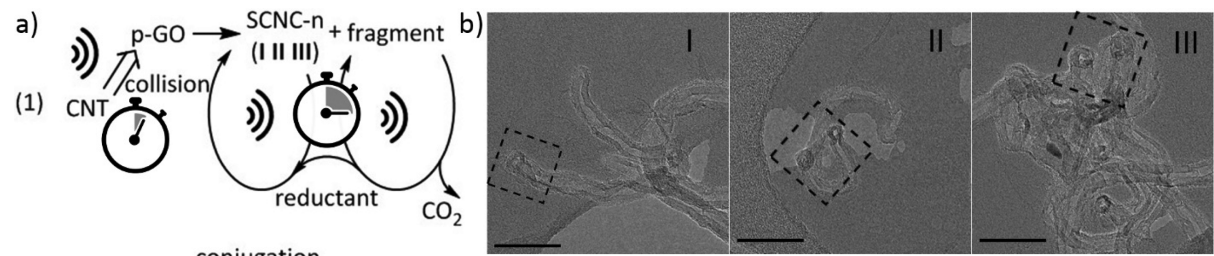

(2) $\mathrm{p}-\mathrm{GO}+\mathrm{CNT} \stackrel{\text { conjugation }}{\longrightarrow} \mathrm{GO}+\mathrm{CNT}$
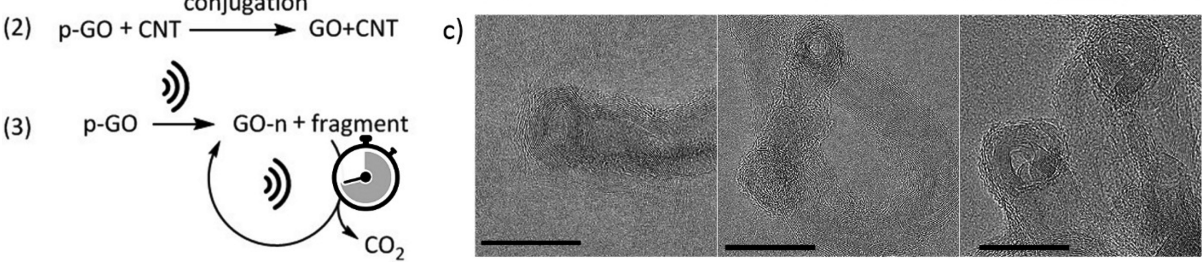

Figure 1. US-assisted formation of GO-CNT covalent bonds. (a) Schematic representation shows the formation procedure of (1) SCNC-n, (2) GO + CNT, and (3) GO-n. The shockwave-induced collision between p-GO and CNT occurs at the earlier stage, which is followed by restoration of graphitic domains. GO $+\mathrm{CNT}$ features with $\pi-\pi$ interactions and GO- $n$ undergo gradual fragmentation, yielding $\mathrm{CO}_{2}$ much later. (b, c) TEM and HRTEM observations of three configurations (I, II, III) of rooting CNTs (red) on GO sheets (blue). Scale bar in (b) and $(\mathrm{c}), 50$ and $20 \mathrm{~nm}$.

method to fabricate graphene-based components with high strain tolerance is rarely reported.

Ultrasonication (US), among the strategies of instantaneous energy input, ${ }^{16,17}$ is a versatile wet method to disperse and produce graphene-based carbon nanomaterials. ${ }^{18-20}$ It is also kinetically complicated due to the intertwined processes, including dispersion/exfoliation or reaggregation, damage, or restoration of crystal structure and radical generation or recombination. Categorically, the vast majority of researchers believe the shockwave-induced $\pi-\pi$ conjugation and lamellar spacing between graphitic domains dominates the superior stretchability and mechanical stability of nanocomposites to their parent materials. ${ }^{15}$ Nevertheless, few examples exist to argue this overtrusted claim in the context that the collisions among suspended solid particles arise during extreme heating at the point of impact to overcome the bonding energy barrier. $^{21,22}$ In a single-component system-graphene oxide $(\mathrm{GO})^{23}$ or carbon nanotube $(\mathrm{CNT})^{24}$ dispersion as a typical model-extensive sonication intensifies the fragmentation and structural damage, while in binary- or even multiplecomponent systems, the understandings of kinetics in bond cleavage, generation of active intermediates and high-velocity heterogeneous collision for bond formation/local fusion are quite vague. $^{25}$

In this work, we carefully distinguish the unusual sonochemical phenomena of a well-known GO-CNT binary system from common concepts of $\pi-\pi$ conjugation and fragmentation and thereby reintroduce US as a reliable method to fabricate a strain-tolerant nanocomposite. Notably, we highlight three critical findings. First, US enables roots-like covalent bonding between GO and CNT, which was only realized before via chemical vapor deposition (CVD). Second, we reveal (1) shockwave-induced collision between pristine GO (p-GO) and CNT ( 0.05 equiv) occurs at the early stage, and the resulting reductant fragments immediately help with restoration of graphitic domains, which is distinct from (2) $\pi-\pi$ conjugative assembly (GO $+\mathrm{CNT})$ and (3) kinetically different from single-component sonication systems (GO-n, CNT- $n$ ) (Figure 1a). Third, covalently bonded CNT induces or retains in-plane compressive stress of crumpled GO sheets, leading to stretchable carbonaceous nanocomposites (SCNC$n$, where $n$ is sonication minutes). The mechano-responsive pattern outperforms $\pi-\pi$ assemblies in retaining electrical conductance and structural integrity even at a strain of $160 \%$.

\section{RESULTS AND DISCUSSION}

Roots-like Covalent Bonding. The additive-free water over organic solvent is employed as sonication medium because water is (1) a well-reported background and versatile reagent in chemical reactions on GO surface, ${ }^{26,27}$ (2) minimization of heteroatomic doping, ${ }^{28}$ and (3) the possibility for environmental-friendly and scalable production. Exclusively for SCNC- $n$, the $\mathrm{pH}$ value increases from 2 to $4-5$, and concurrently, the zeta-potential decreases from -12.5 to -8.8 $\mathrm{mV}$. The estimated bandgap energy from light extinction is minimized at SCNC-15 $(\sim 3.5 \mathrm{eV})$, while GO-15 and GO + CNT change little in comparison with p-GO $(\sim 4.35 \mathrm{eV})$. Wide-scan survey X-ray photoelectron spectroscopy (XPS) further showed a much higher $\mathrm{C} / \mathrm{O}$ atomic ratio of SCNC-15 (4.9) than p-GO (2.2), GO-15 (2.0), and GO + CNT (2.6), suggesting a notable removal of oxygen-containing groups during US (Table S1 and Figures S1 and S2). All of the above preliminary results strongly indicate that ultrasound plays a role not only in breaking up the entanglement and guiding conjugative assembly ${ }^{19,29}$ but also in enabling mechanochemical reactions yet to be clarified.

TEM images of SCNC-15 (Figure 1b) reveal three configurations of the anchoring CNTs, including single concentric root (I), two or multiple roots per CNT (II), and multiple roots belonging to an entanglement (III). A statistic of CNTs (and entanglement) presents an averaged frequency of appearance of I, II, and III, which is approximately $24 \%$, $35 \%$, and $12 \%$. The proportion ratio of I and II varies in each experiment, but III can be reproducibly minimized to zero until SCNC-30. The magnified images (Figure 1c) unveil that the outermost layers of CNT roots are disrupted and gradually merged with GO planes. These distorted carbon lattices imply the out-of-plane bonding region, which is similar to the pillared graphene/CNT junctions induced by $\mathrm{CVD} .{ }^{25,30-35}$ In addition, we approved the robustness of our roots-like covalent bonding over the $\pi-\pi$ interactions that could also expose the open ends of CNTs (see Figures S3-S6).

We also exclude the possibility that I, II, and III are the free open ends of unzipped CNT with sidewalls melded onto GO. ${ }^{36}$ Shell-isolated nanoparticle-enhanced Raman spectros- 
copy (SHINERS $)^{37}$ of GO-15 is featureless, and a CNT-15 or CNT-15 + GO-15 physical mixture shows three companion modes at around 1170, 1255, and $1450 \mathrm{~cm}^{-1}$ which are assigned to the vibration mode of transpolyacetylene-like chains at the open concentric edges of $\mathrm{CNTs}^{38-40}$ (see the Methods and Figure 2a). However, such modes are negligible

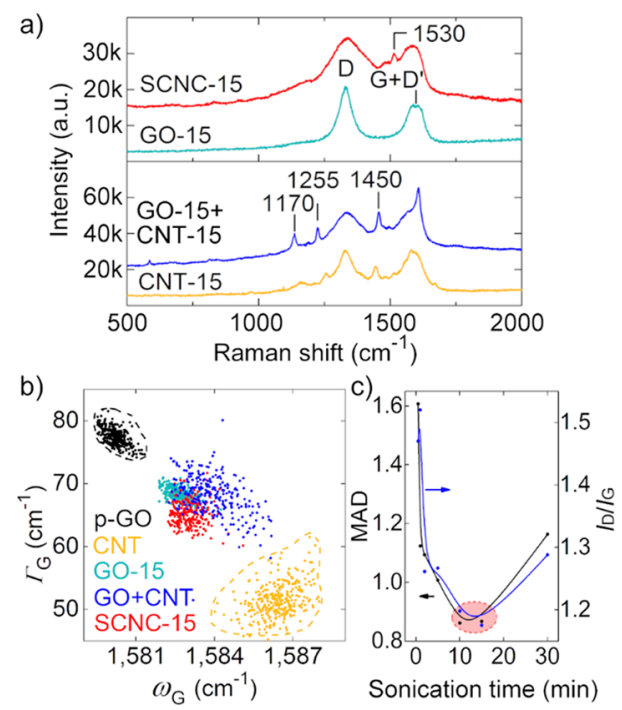

Figure 2. Analysis of Raman spectra. (a) SHINERS on SCNC-15, GO-15, CNT-15, and GO-15+CNT-15. Normal Raman spectrum consists of three peaks: D band $\left(\sim 1350 \mathrm{~cm}^{-1}\right)$, G band $(\sim 1583$ $\left.\mathrm{cm}^{-1}\right)$, and $\mathrm{D}^{\prime}$-bands $\left(\sim 1620 \mathrm{~cm}^{-1}\right)$. (b) $\Gamma_{\mathrm{G}}$ versus $\omega_{\mathrm{G}}$ recorded on spots on regions of p-GO (black), CNT (orange), GO-15 (dark cyan), GO + CNT (blue), and SCNC-15 (red). (c) MAD and $I_{\mathrm{D}} / I_{\mathrm{G}}$ as functions of sonication time for SCNC- $n$.

for SCNC-15. Instead, SCNC-15 is featured with a mode at $1530 \mathrm{~cm}^{-1}$ which refers to $\mathrm{C}\left(\mathrm{sp}^{3}\right)$-aryl bonds or five- and seven-membered carbon rings in the regions with high local curvature. ${ }^{41}$ This result further supports the previous suggestion based on our TEM observations.

Sonochemical Assembly. We then carried out of spatially resolved Raman analysis to set SCNC apart from $\pi-\pi$ assembly. Figures $2 \mathrm{~b}$ and $\mathrm{S} 7$ show the spectra of $\mathrm{p}-\mathrm{GO}$ (black) differentiate CNT (orange) with the lower frequency of the $\mathrm{G}$ band $\left(\omega_{\mathrm{G}}\right)$ and wider full width at half-maximum (fwhm, here $\Gamma_{\mathrm{G}}$ ). The difference among GO-15 (cyan), SCNC-15 (red), and GO + CNT (red) becomes evident when we compare the mean absolute deviation (MAD) of points of each sample (see the Methods). The MAD order is p-GO $\approx$ GO-15 $(0.47)<\operatorname{SCNC}-15(0.86)<\mathrm{GO}+\mathrm{CNT}(2.35) \approx$ CNT. Even though SCNC-15 and GO + CNT are analogous at a micron level (Figure S8), the comparison of MAD can be a potential spectral methodology to differentiate much more homogeneous sonochemical hybridization from the conjugative assembly.

Parallel to $\mathrm{MAD}$, we take the averaged $\mathrm{D} / \mathrm{G}$ intensity ratio $\left(I_{\mathrm{D}} / I_{\mathrm{G}}\right)$ reflecting variations in defective and graphitic domains of SCNC- $n .^{42}$ An identical temporal-dependent process was found: both MAD and $I_{\mathrm{D}} / I_{\mathrm{G}}$ decrease sharply at the initial 5 min and reach the lowest value at between SCNC-10 and 15 . $I_{\mathrm{D}} / I_{\mathrm{G}}$ of SCNC-15 is even lower than GO-15 (1.32), GO + CNT (1.21), p-GO (1.20), and CNT (1.19) (Figure 2C and Figure S9). Thus, the US-induced assembly accompanies the restoration of $\mathrm{sp}^{2}$ carbon networks, rather than only conjugative adhesion of CNT. These coupled processes enable us to clarify the mechanism behind US-induced hybridization by interpreting the restoration of graphitic domains in SCNC$n$.

Deconvoluted XPS C 1s spectra of SCNC-15 unveil $\sim 10$ atom $\%$ increase for both $\mathrm{sp}^{2}$ - and $\mathrm{sp}^{3}$-hybridized carbon compared with p-GO and GO-15. Elimination of $\mathrm{C}-\mathrm{O}-\mathrm{C}$ and $\mathrm{O}-\mathrm{C}=\mathrm{O}$ contributes to the major diminishment of oxygen, but $\mathrm{C}-\mathrm{OH}$ surprisingly increases from 4.8 to 19.6 atom \% (Figure 3a). Furthermore, FTIR temporal analysis of SCNC-n even illustrates that the overall evolution of oxygen-containing groups achieves equilibrium in only $1 \mathrm{~min}$, leaving more stable
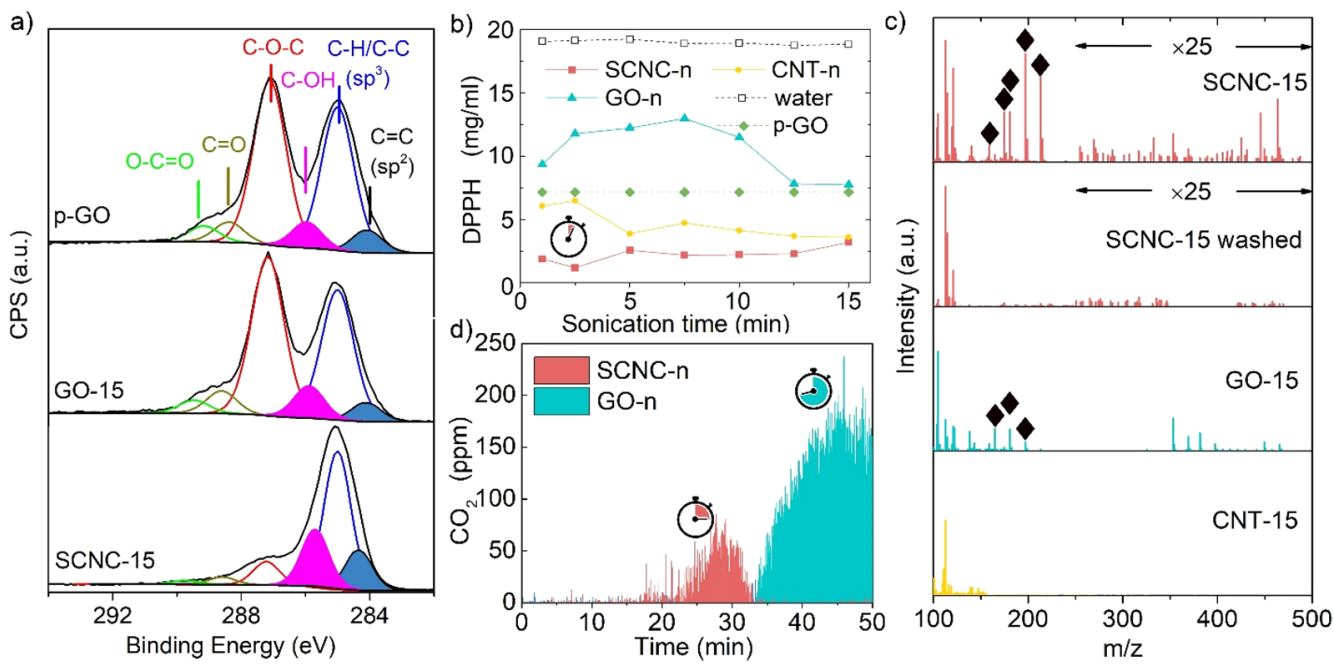

Figure 3. Clarification of the mechanism of US-assisted assembly of GO and CNT. (a) XPS C 1s spectra of p-GO, GO-15, and SCNC-15 reveal a dramatic difference in evolution of functional groups. (b) Remaining DPPH after reaction with each sonicated mixture was checked. System of SCNC- $n$ quenches the characteristic absorption more than that of water, GO- $n$, and CNT- $n$ during the whole sonication process, with the absorption of $\mathrm{p}$-GO as reference. (c) MALDI-TOF mass spectrum of small-molecular fragments of SCNC-15 containing some of reported ( ) flavonoid antioxidant. (d) Detection of $\mathrm{CO}_{2}$ during the ultrasonication unveiled that the deoxygenation in SCNC-n system is faster and less severe than that of GO- $n$. 


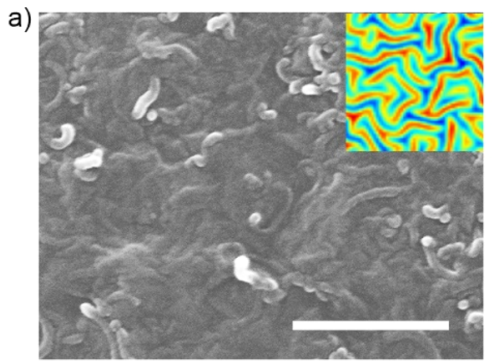

d)

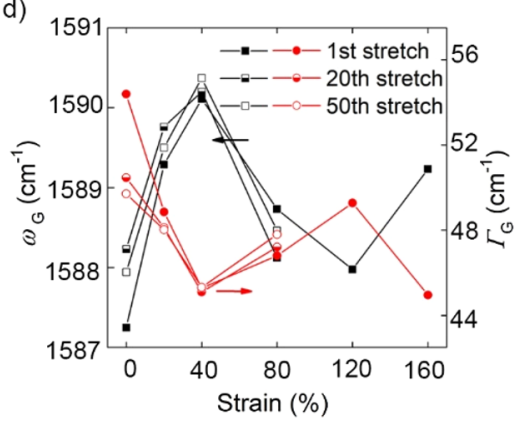

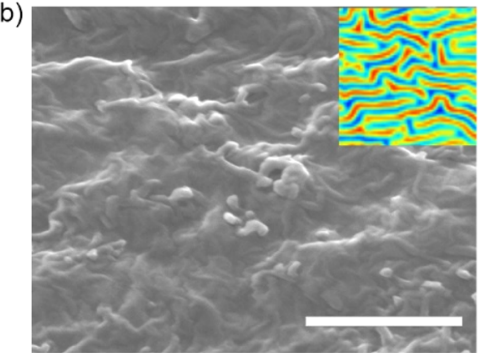
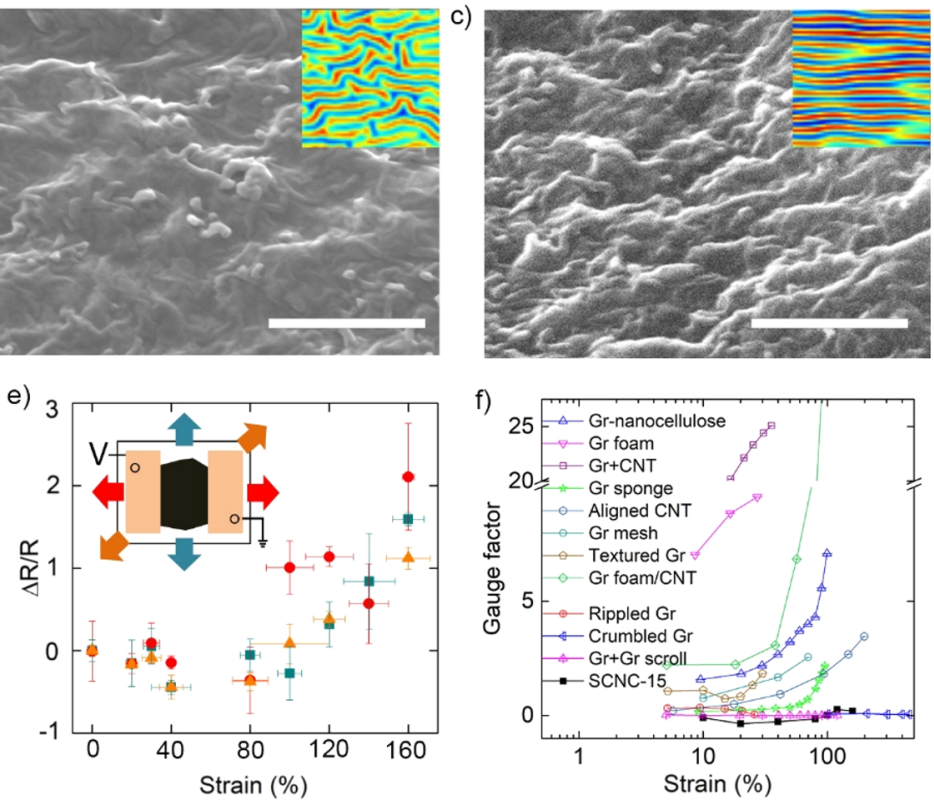

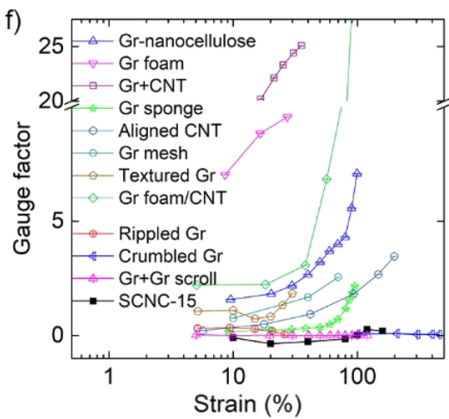

Figure 4. Strain-tolerant electrical conductance and structural integrity. (a-c) SEM images of SCNC-15 flakes deposited on a PDMS substrate which was stretched by a precise vice at strain of 0,40 , and $120 \%$, respectively (scale bar $1 \mu \mathrm{m}$ ). Insets are snapshots of an ideal $2 \mathrm{D}$ flake which was simulated to be isometrically compressed into irregular wrinkles, stretched uniaxially and fully stretched. (d) $\Gamma_{\mathrm{G}}$ and $\omega_{\mathrm{G}}$ of SCNC-15 as functions of strain are shown, along with the variations at 20th and 50th stretch. (e) Upon being stretched at transverse (red), longitudinal (dark cyan), and diagonal (orange) directions, the resistance of SCNC-15 in each case shows the initial decrease before the strain of $80 \%$ and slight increase until $160 \%$. The inset shows the schematic of our strain-conductivity measurement setup, with flakes bridging two Pt electrodes, across which the scanning voltage from -0.4 to $0.4 \mathrm{~V}$ was applied. (f) Gauge factors of different stretchable carbonaceous materials/elastomer composites as functions of strain are shown. Graphene is referred to as Gr.

hydroxyl and ether groups (see Figure S10). It first rules out the role of ultrasonic thermal deoxygenation that removes $-\mathrm{OH}$ by instantaneous hot spot or local thermal annealing. ${ }^{43-45}$ The main origin of $-\mathrm{OH}$ is deemed to be nucleophilic attack of $\mathrm{H}_{2} \mathrm{O}$ to surface vacancies and edge dangling bonds generated right after bond cleavage or decarboxylation in the aqueous medium. ${ }^{46}$ Meanwhile, sonolysis of water primarily produces reactive hydrogen and hydroxyl radicals $\left(\mathrm{H}_{2} \mathrm{O} \rightarrow{ }^{\circ} \mathrm{H}+{ }^{\bullet} \mathrm{OH}\right),{ }^{28}$ and $-\mathrm{OH}$ groups can consume oxidative radicals $\left({ }^{\circ} \mathrm{OH}\right)$ to prevent the radicals' recombination or produce ${ }^{\bullet} \mathrm{H}\left({ }^{\bullet} \mathrm{OH}+\mathrm{OH}=\mathrm{H}_{2} \mathrm{O}+{ }^{\bullet} \mathrm{H}\right) .^{46}$ The variation of ${ }^{\bullet} \mathrm{H}$ radicals, therefore, illustrates the evolution of $-\mathrm{OH}$-loaded moieties. We conduct the DPPH assay to quantify the $\mathrm{H}$-donator (see the Supporting Information, Figure $3 b$, and Figure S11). As expected, SCNC- $n$ notably quenches the DPPH absorbance (at $516 \mathrm{~nm}$ ) faster than GO- $n$ or CNT- $n$ from the beginning of sonication to the end. The $\mathrm{H}$ donator reaches the highest concentration at the first $2.5 \mathrm{~min}$ under US, which represents the early increase of $-\mathrm{OH}$ groups and also coincides with the considerable decrease of $I_{\mathrm{D}} / I_{\mathrm{G}}$ from SCNC-1 (1.52) to SCNC-5 (1.27).

We indeed found $-\mathrm{OH}$-containing fragments, small $(0-4 \mathrm{k}$ $\mathrm{Da})$ and large $(5-50 \mathrm{k} \mathrm{Da})$, absorbed on the surface of SCNC15 by using matrix-assisted laser desorption ionization time-offlight mass spectrometry (MALDI-TOF MS). Figure $3 c$ even shows some recognized fragments of flavonoid-type molecules, mainly derived from the stepwise loss of $\mathrm{CO} / \mathrm{CO}_{2}$, at $m / z 241$, 213, 197, and 181 (Figure S12). ${ }^{47}$ Note that all of the adsorbates can be washed away with isopropyl alcohol or acetonitrile, confirming their noncovalent connections with SCNC- $n$ surface. The generation of $\mathrm{CO}_{2}$ as a final product of deoxygenation was tracked to confirm the proposed mechanism (see the Methods, Figure 3d, and Figure S13). We found
SCNC- $n$ increased, maximized, and ceased the $\mathrm{CO}_{2}$ concentration in carrier flow just before $15 \mathrm{~min}$ sonication (30 $\mathrm{min}$ with $5 \mathrm{~s}$ on and $5 \mathrm{~s}$ off), whereas GO- $n$ exhibited a much lagged but massive $\mathrm{CO}_{2}$ production. The kinetics of $\mathrm{CO}_{2}$ production of SCNC-n matches the $5 \mathrm{~min}$ window we found for the optimization of $\mathrm{MAD}$ and $I_{\mathrm{D}} / I_{\mathrm{G}}$.

As such, a convincing explanation of the restoration of graphitic domains is that the shockwaves arise to high-velocity GO-CNT collisions, inducing GO-CNT covalent bonds and fast generation of fragments (Figure 1a). CNT is more likely to be rooted at the edge or a defective basal plane of GO at the initial stage of collision, as we found in SCNC-5 (Figure S14) and other work. ${ }^{19}$ The free fragments increase in a short time but decrease gradually as less probability of GO-CNT collision. The initial fragments continue alternatively to interact with ${ }^{\bullet} \mathrm{OH}$ and further fragment, yielding carbonyls or carboxylates for deoxygenation and more stable hydroxyl/ ether loading fragments. ${ }^{46}$ In the presence of ${ }^{\bullet} \mathrm{OH}$ and $\mathrm{H}_{2} \mathrm{O}$, carbonyls and carboxylates will be easily converted into gaseous $\mathrm{CO} / \mathrm{CO}_{2}$ or dissolved $\mathrm{H}_{2} \mathrm{CO}_{3}$ to increase the $\mathrm{pH}$ of the system. The unpaired electrons lead to the formation of covalent bonds with $\mathrm{H}_{2} \mathrm{O}$ or other carbon atoms. ${ }^{48}$ Unlike gradually fragmented GO- $n$, the burst increase of surfaceabsorbed fragments at the initial stage of sonication protects SCNC- $n$ from intensive fracture, leading to flakes twice the size of GO- $n$ (Table S1). When the fragments are used up, SCNC30 increases $\mathrm{MAD}$ and $I_{\mathrm{D}} / I_{\mathrm{G}}$ (Figure $2 \mathrm{c}$ ). Without initial collision and fragmentation, GO + CNT under US for extra 15 min keeps MAD unchanged, but $I_{\mathrm{D}} / I_{\mathrm{G}}$ increases from 1.21 to 1.31. Furthermore, MALDI-TOF MS detected a similar fragmentation when GO and fullerene were cosonicated (Figure S15), implying that the mechanism of collision can also be applied to other binary-component systems. We also 
find that excess fullerene proportion intensifies the collision frequency and degrades the MAD and $I_{\mathrm{D}} / I_{\mathrm{G}}$, reflecting the possible detrimental effect of collision (Figure S16). In addition, we also discussed the size and dimension of the secondary carbonaceous component (see the Supporting Information and Figure S17). We found the large 3D objects weakened the collision, ${ }^{21,22}$ while a $2 \mathrm{D}$ partner led to a homogeneous assembly mainly by $\pi-\pi$ conjugation or hydrophobic attraction.

Strain-Tolerant Structural Integrity and Conductance. Morphologically, the crumples and rooting CNTs are densely observed on SCNC-15 (Figure 4a). It is difficult to decouple the effects of rooting CNTs and heterogeneous atomic hybridization of $\mathrm{sp}^{2}$ networks to the out-of-plane deformation. ${ }^{49,50}$ The rooting CNTs indeed retain the crumples against $2 \mathrm{D}$ capillary-force stretching during drying on a substrate, whereas $\mathrm{p}-\mathrm{GO}, \mathrm{GO}-n$, and GO + CNT are flattened easily (Figure S18). The mechano-response becomes notable as the tensile strain increases to $40 \%$ uniaxially. The regular ridges along the stretching direction can be identified (Figure $4 \mathrm{~b}$ ). Until the strain reaches $120 \%$, all nanotubes were extensively stretched and merged with the ridges and, if present, grooves (Figure 4c). The ridges tend to align along the tensile direction with a roughly averaged distance of 300 $\mathrm{nm}$, although they are slightly distracted locally by the innate stress. Our parallel simulations reveal the configurations II and III of rooting CNTs can induce in-plane expansion of the SCNC-15, which is in turn constrained by the substrate, engendering compressive stresses in SCNC-15. When the SCNC-15 substrate is stretched, the nanotubes are extended along the tensile direction, and in turn, the stresses between CNTs and GO also distribute along the tensile direction (insets of Figure $4 a-c$, see the Supporting Information). The variation from labyrinthine crumpling to a groove pattern with the magnificence of strain is consistent with our SEM observation.

Spectrally, the stretched SCNC-15 shows $\sim 3 \mathrm{~cm}^{-1}$ blueshifts of $\omega_{\mathrm{G}}$ and $10 \mathrm{~cm}^{-1} \Gamma_{\mathrm{G}}$ narrowings of $\mathrm{G}$ peak below $40 \%$ strain, followed by the stress-induced redshifts of $\omega_{\mathrm{G}}$ (by $\sim 2 \mathrm{~cm}^{-1}$ ) and increases of $\Gamma_{\mathrm{G}}$ (by $\sim 4 \mathrm{~cm}^{-1}$ ) at the strain of $120 \% .^{51,52}$ Such "relax-stress" alteration of SCNC-15 generally maintains good reproducibility when the substrate is repeatedly stretched to the $80 \%$ strain and back (Figure $4 \mathrm{~d}$ ). In comparison, the conventional 2D flakes usually undergo an opposite "stressrelax" or even cracking process under tensile strain, ${ }^{53}$ and so we found for p-GO, GO-15, and GO + CNT (Figure S19S21). Inspired by the Raman "relax-stress" alteration of SCNC15 and strong electron-phonon coupling of graphene-based materials, ${ }^{54}$ we deduce that stress-induced variations of electronic structure can be relieved by counterweighing between the intrinsic and external strains.

Without any reduction, the electrical conductivity of SCNC15 flakes is estimated at a range of $0.46-1.84 \times 10^{4} \mathrm{~S} / \mathrm{m}$, already approaching the record of reduced GO monolayer (2.0 $\left.\times 10^{4} \mathrm{~S} / \mathrm{m}\right)^{55}$ (see the Methods and Figure S22). When the strain is below $80 \%$, the resistance $(R)$ remains almost unchanged $\left(\Delta R=R-R_{0} \approx 0, R_{0}\right.$ defined as the resistance without strain) at transverse, longitudinal, and diagonal directions (Figure 4e). It indicates that the multidirectional rooting CNTs maintain an efficient connection across SCNC15 to overcome the transport barriers. Beyond bridging the sheets, the rooting CNTs restore the structural integrity of SCNC-15 even at a strain of $160 \%$ (Figure S23). It is in contrast to the conventional pillared structures that undermine the overall tensile strength because of localized high stress concentration. $^{56}$ The propagation of microcracks is ceased, as evidenced by the fact that the resistance of SCNC-15 remains almost intact while GO + CNT has a 50000-fold increase (Figure S24). The strain tolerance was evaluated by comparing the gauge factor (defined by $\Delta R /(R \times$ strain $))$ of SCNC-15 with the existing stretchable nanocarbonaceous composites. Regarding the gauge factor (0.06) and strain durability (0$160 \%)$, the single-layer SCNC-15 is analogous to the bestperforming crumbled graphene $\mathrm{e}^{10}$ and graphene scroll hybrid ${ }^{12}$ (Figure $4 \mathrm{f}$ and Table S2). These properties make SCNC-15 particularly suitable for flexible electronic devices.

\section{CONCLUSIONS}

To summarize, we confirm that US induces roots-like covalent bonds by carefully distinguishing the GO-CNT binarycomponent system from the single ones and the pairs dominated by $\pi-\pi$ conjugative assembly. The US-directed assembly was systematically tracked to produce nanocomposites whose electrical conductance resists high external strain by its mechano-responsive crumbled structure. The rooting morphologies of CNTs reflect the covalent bonding formed by shockwave-mediated collisions between GO and CNT. Adopting the collision strategy can not only guide the formation of all-carbon composites but also provides an approach to understand the concurrence of restoration of $\mathrm{sp}^{2}$ networks and homogeneous assembly in the binary-component sonication systems between $\mathrm{GO}$ and $\mathrm{Au}, \mathrm{Ag}, \mathrm{Pt}, \mathrm{Pd}, \mathrm{ZnO}$, $\mathrm{TiO}_{2}$, and $\mathrm{CuO}$ nanoparticles. ${ }^{43}$

Although we confirmed the covalent bonding and securitized the role of US in making stretchable composites, it is unclear yet how the fragments and rooting CNT affect the mechanical and electrical properties of planar materials. To address this problem, we are trying to couple the PeakForce with the conductive AFM techniques. To fundamentally understand the process of collision between carbon materials $(0.1-100 \mathrm{~km} / \mathrm{s}),{ }^{57-59}$ the dependence of collision velocity on the size and dimensions need to be quantified. Our current sonication model only partially explains these results. Determination of the exact mechanism and precise tailoring collision kinetics for desired performance will be the focus of future work.

\section{MATERIAL AND METHODS}

Fabrication of SCNC- $n$ and $\mathrm{CO}_{2}$ Sensing. We conducted US protected by argon within a Suslick cell to discount the disruptive reactions (Figure S13). Before use, all of the GO solution $(2 \mathrm{mg} / \mathrm{mL})$, multiwalled CNT (O.D. $\times L, 6-13 \mathrm{~nm} \times 2.5-20 \mu \mathrm{m})$, and fullerene from Sigma-Aldrich were washed with IPA three times. The washed GO was redispersed in water. CNT and C60 solids were dried under vacuum. The mixture of $8 \mathrm{~mL}$ of $\mathrm{GO}$ aqueous solution $(2 \mathrm{mg} / \mathrm{mL}, \mathrm{pH}$ $=2$ ) and $0.8 \mathrm{mg}$ of CNT was pulse-sonicated (model FB705, $20 \mathrm{kHz}$, Power 700 W, Fisher Scientific, USA) for a certain time (amplitude: $40 \%, 5 \mathrm{~s}$ on and $5 \mathrm{~s}$ off) with a titanium microtip probe $(1 / 8 \mathrm{in}$.) in the Suslick reaction cell (cooled by $4{ }^{\circ} \mathrm{C}$ circulating water). Then the power density at probe surface was calculated to be around $216 \mathrm{~W} /$ $\mathrm{cm}^{2}$. Sonication of $\mathrm{GO} / \mathrm{C} 60, \mathrm{GO}$, or CNT followed the same procedure. SprintIR-W high-speed $\mathrm{CO}_{2}$ sensor with a flow adapter was connected to the gas outlet of Suslick cell. The sensor takes record 20/s. Here, we controlled the argon rate as 3-4 bubbles/ second.

Raman Analysis. For SHINERS, Reinshaw 50LWD objective was used. The excitation wavelength was $637.8 \mathrm{~nm}$ from a $\mathrm{He}-\mathrm{Ne}$ laser 
(power is about $1 \mathrm{~mW}$ ). Raman frequencies were calibrated using $\mathrm{Si}$ wafer spectra. The Raman spectra shown in the experiment were collected during $60 \mathrm{~s}$ for one single spectrum curve one time. Arrays of $55 \mathrm{~nm}$ gold nanoparticle with 3-4 nm silica shell covered the sample surface which was rinsed by IPA before gold coverage. Raman mapping and spectra were acquired with a LabRam Xplora confocal Raman microscope equipped with a confocal microscope and a motorized $x-y-z$ stage. Measurements were conducted with $50 \times$ long working distance objective lens, and a linear polarized laser $(\lambda=$ $532 \mathrm{~nm}$ ) excitation was focused with a diffraction-limited spot size (theoretical 1.22 $\lambda / \mathrm{NA}$ ) on samples. The Raman signal was detected with a grating 1800 grooves $\mathrm{mm}^{-1}$, along with a $1 \%$ filter, $200 \mu \mathrm{m}$ slit, and $500 \mu \mathrm{m}$ hole. For mapping, $1 \mu \mathrm{m}$ steps were chosen, and every pixel corresponds to one scan. The spectrum from each location was obtained by averaging $3 \mathrm{~s}$ cycles. We characterized each Raman peak ( $\mathrm{G}, \mathrm{D}$, and $\mathrm{D}^{\prime}$ ) by Lorentzian fit, and we fit those peaks together using a double-Lorentzian fit. The mean absolute deviation (MAD) of a data set is the average distance between each data value and the mean. The mean absolute deviation describes variation in a data set. The MAD of a set $\left\{\omega_{\mathrm{G} 1}, \Gamma_{\mathrm{G} 1} ; \omega_{\mathrm{G} 2}, \Gamma_{\mathrm{G} 2} ; \omega_{\mathrm{G} 3}, \Gamma_{\mathrm{G} 3} ; \ldots ; \omega_{\mathrm{Gn}}, \Gamma_{\mathrm{Gn}}\right\}$ is

$$
\frac{1}{n} \sum_{i=1}^{n} \sqrt{\left(\omega_{\mathrm{Gn}}-\overline{\omega_{\mathrm{G}}}\right)^{2}+\left(\Gamma_{\mathrm{Gn}}-\overline{\Gamma_{\mathrm{G}}}\right)^{2}}
$$

where $\overline{\omega_{\mathrm{G}}}$ and $\overline{\Gamma_{\mathrm{G}}}$ are the mean value of $\omega_{\mathrm{G}}$ and $\Gamma_{\mathrm{G}}$, respectively.

Stretchability and Conductivity. The casting solution was prepared by diluting the dispersion 25 times with deionized water. A $50 \mu \mathrm{L}$ portion of the solution was cast onto a flat glass or an elastic PDMS substrate, which was dried under vacuum overnight. Then, two Pt electrodes with a thickness of $60 \mathrm{~nm}$ were deposited as the metal contacts on flakes via a shadow mask by radiofrequency sputtering using a Moorfield nanoPVD-S10A system at $45 \mathrm{~W}, 3 \times 10^{-4} \mathrm{mbar}$ in pure Argon. The gap spacing between two Pt electrodes was $20 \mu \mathrm{m}$. Elastic PDMS substrates were prepared by spin-coating Sylgard 184 on a clean silicon wafer. The films were then cured at $100^{\circ} \mathrm{C}$ for $3 \mathrm{~h}$ and peeled off ( $0.5 \mathrm{~mm}$ in thickness). The method to measure the resistance of a sample is the two-probe technique that consists of contacting two probes and measuring the voltage while a current is applied between the probes. The samples were fixed to the surface of small vice clamp with superglue. The strain was applied by turning the knob of vice. Repeatability characteristics have been performed by the handle control mechanism. The strain of PDMS was determined by two reference dots close to the measured flake samples. Unless otherwise stated, all of the strains mentioned in this work are determined by the reference dots on PDMS.

MALDI-TOF MS Analysis. The original $2 \mathrm{mg} / \mathrm{mL}$ dispersion was first diluted to $0.5 \mathrm{mg} / \mathrm{mL}$ with water and then mixed in methanol for $50 \% \mathrm{v} / \mathrm{v}$. After being shaken for $1 \mathrm{~min}, 2.5 \mu \mathrm{L}$ of the suspension was pipetted onto the MTP 384 ground steel target. The sample target was left at room temperature for 10-15 $\mathrm{min}$ for evaporation of the solvent. For washed SCNC-15, the original dispersion was added to 2propanol $(1: 10)$ to form a flocculent suspension. After centrifugation at $8000 \mathrm{rpm}$ for $15 \mathrm{~min}$, the supernatant was removed, and the pellet was washed with IPA two more times and then dispersed in water. The autoflex maX MALDI-TOF MS system (Bruker) was utilized with the mass-to-charge ratio $(\mathrm{m} / \mathrm{z})$ range of $0-4 \mathrm{k}$ and 5-50k Da. The methods of RP for low mass range and LP Da for medium mass range were chosen. Fifty shots at raster spot, 10000 shots, frequency of 2000 , and intensity of $55 \%$ were adopted for measurement. Raw mass spectra were exported as text files (.txt) using flexAnalysis software. All subsequent data analyses were conducted with methods of $0-3.5 \mathrm{k} \mathrm{Da}$ and $5-20 \mathrm{k} \mathrm{Da}$ for spectral pattern matching. Peaks with a signal-to-noise ratio $(\mathrm{S} / \mathrm{N})$ of at least three were extracted from each spectrum after baseline correction and intensity normalization.

\section{ASSOCIATED CONTENT}

\section{S Supporting Information}

The Supporting Information is available free of charge on the ACS Publications website at DOI: 10.1021/acsnano.9b06366.
General methods, supporting table (Tables S1 and S2), and detailed spectra and experimental data (Figures S1S23) (PDF)

\section{AUTHOR INFORMATION}

\section{Corresponding Author}

*E-mail: d.shchukin@liverpool.ac.uk.

ORCID

Bo Li: 0000-0002-3792-2469

Jian-Feng Li: 0000-0003-1598-6856

Dmitry G. Shchukin: 0000-0002-2936-804X

\section{Present Address}

\#(Z.Z.) Max Planck Institute for Polymer Research, Ackermannweg 10, 55128 Mainz, Germany.

\section{Author Contributions}

Z.Z. conceived and performed most of the experiments, interpreted the data, and wrote the manuscript; J.J. contributed in the $I-V$ measurement and comprehension of electrical conductivity. B.L. and G.-K.X. helped conduct and understand simulation of the crumpled structure and strain-dependent variation of the crumples. J.-C.D. and J.-F.L. supported with operation on SHINERS. D.S. supervised the complementation of the project.

\section{Notes}

The authors declare no competing financial interest.

\section{ACKNOWLEDGMENTS}

Z.Z. and D.S. acknowledge finance support from ERC Consolidator and Proof-of-Concept Grants (ERC 647969, 767173). Z.Z. acknowledges a Marie Sklodowska-Curie individual Fellowships Grant (796927). Dr. Ming Liu and Dr. Steven Robinson from the Materials Innovation Factory in UoL are thanked for their inspiration and help with MALDIToF MS. Z.Z. thanks Jianli Zou, Tobias Heil, and Evanthia Adamidou for help with AFM, TEM, and DLS. Z.Z. and D.S. sincerely acknowledge Prof. Helmuth Moehwald who passed away March 27, 2018. We thank him for his open way with young people and great support for their career development. In particular, this work is dedicated to him in recognition of his world-leading role in sonochemistry. D.S. acknowledges financial support from the Russian Science Foundation (Grant No. 19-79-30091) provided for surface structure measurements.

\section{REFERENCES}

(1) Trung, T. Q.; Lee, N.-E. Recent Progress on Stretchable Electronic Devices with Intrinsically Stretchable Components. Adv. Mater. 2017, 29, 1603167.

(2) Rogers, J. A.; Someya, T.; Huang, Y. Materials and Mechanics for Stretchable Electronics. Science 2010, 327, 1603-1607.

(3) Lee, C.; Wei, X.; Kysar, J. W.; Hone, J. Measurement of The Elastic Properties and Intrinsic Strength of Monolayer Graphene. Science 2008, 321, 385-388.

(4) Jiang, J.-W.; Chang, T.; Guo, X.; Park, H. S. Intrinsic Negative Poisson's Ratio for Single-Layer Graphene. Nano Lett. 2016, 16, $5286-5290$

(5) Lee, S.-K.; Kim, B. J.; Jang, H.; Yoon, S. C.; Lee, C.; Hong, B. H.; Rogers, J. A.; Cho, J. H.; Ahn, J.-H. Stretchable Graphene Transistors with Printed Dielectrics and Gate Electrodes. Nano Lett. 2011, 11, $4642-4646$

(6) Jang, H.; Park, Y. J.; Chen, X.; Das, T.; Kim, M.-S.; Ahn, J.-H. Graphene-Based Flexible and Stretchable Electronics. Adv. Mater. 2016, 28, 4184-4202. 
(7) Tao, P.; Shang, W.; Song, C.; Shen, Q.; Zhang, F.; Luo, Z.; Yi, N.; Zhang, D.; Deng, T. Bioinspired Engineering of Thermal Materials. Adv. Mater. 2015, 27, 428-463.

(8) Yan, W.; He, W.-Y.; Chu, Z.-D.; Liu, M.; Meng, L.; Dou, R.-F.; Zhang, Y.; Liu, Z.; Nie, J.-C.; He, L. Strain and Curvature Induced Evolution of Electronic Band Structures in Twisted Graphene Bilayer. Nat. Commun. 2013, 4, 2159.

(9) Grima, J. N.; Winczewski, S.; Mizzi, L.; Grech, M. C.; Cauchi, R.; Gatt, R.; Attard, D.; Wojciechowski, K. W.; Rybicki, J. Tailoring Graphene to Achieve Negative Poisson's Ratio Properties. Adv. Mater. 2015, 27, 1455-1459.

(10) Zang, J.; Ryu, S.; Pugno, N.; Wang, Q.; Tu, Q.; Buehler, M. J.; Zhao, X. Multifunctionality and Control of The Crumpling and Unfolding of Large-Area Graphene. Nat. Mater. 2013, 12, 321-325.

(11) Blees, M. K.; Barnard, A. W.; Rose, P. A.; Roberts, S. P.; McGill, K. L.; Huang, P. Y.; Ruyack, A. R.; Kevek, J. W.; Kobrin, B.; Muller, D. A.; McEuen, P. L. Graphene Kirigami. Nature 2015, 524, 204-207.

(12) Liu, N.; Chortos, A.; Lei, T.; Jin, L.; Kim, T. R.; Bae, W.-G.; Zhu, C.; Wang, S.; Pfattner, R.; Chen, X.; Sinclair, R.; Bao, Z. Ultratransparent and Stretchable Graphene Electrodes. Sci. Adv. 2017, 3, e170015.

(13) Han, J.; Lee, J. Y.; Lee, J.; Yeo, J. S. Highly Stretchable and Reliable, Transparent and Conductive Entangled Graphene Mesh Networks. Adv. Mater. 2018, 30, 1704626.

(14) Yan, C.; Wang, J.; Kang, W.; Cui, M.; Wang, X.; Foo, C. Y.; Chee, K. J.; Lee, P. S. Highly Stretchable Piezoresistive GrapheneNanocellulose Nanopaper for Strain Sensors. Adv. Mater. 2014, 26, 2022-2027.

(15) Guo, F.; Jiang, Y.; Xu, Z.; Xiao, Y.; Fang, B.; Liu, Y.; Gao, W.; Zhao, P.; Wang, H.; Gao, C. Highly Stretchable Carbon Aerogels. Nat. Commun. 2018, 9, 881.

(16) Guo, P.; Yang, X.; Ye, Q.; Zhang, J.; Wang, H.; Yu, H.; Zhao, W.; Liu, C.; Yang, H.; Wang, H. Laser-Generated Nanocrystals in Perovskite: Universal Embedding of Ligand-Free and Sub-10 nm Nanocrystals in Solution-Processed Metal Halide Perovskite Films for Effectively Modulated Optoelectronic Performance. Adv. Energy Mater. 2019, 1901341.

(17) Jian, J.; Xu, Y.; Yang, X.; Liu, W.; Fu, M.; Yu, H.; Xu, F.; Feng, F.; Jia, L.; Friedrich, D.; Van De Krol, R.; Wang, H. Embedding Laser Generated Nanocrystals in $\mathrm{BiVO}_{4}$ Photoanode for Efficient Photoelectrochemical Water Splitting. Nat. Commun. 2019, 10, 2609.

(18) Yu, X.; Cheng, H.; Zhang, M.; Zhao, Y.; Qu, L.; Shi, G. Graphene-Based Smart Materials. Nat. Rev. Mater. 2017, 2, 17046.

(19) Shin, M. K.; Lee, B.; Kim, S. H.; Lee, J. A.; Spinks, G. M.; Gambhir, S.; Wallace, G. G.; Kozlov, M. E.; Baughman, R. H.; Kim, S. J. Synergistic Toughening of Composite Fibres by Self-Alignment of Reduced Graphene Oxide and Carbon Banotubes. Nat. Commun. 2012, 3, 650 .

(20) Chen, M. Q.; Guan, R. N.; Yang, S. F. Hybrids of Fullerenes and 2D Nanomaterials. Adv. Sci. 2019, 6, 1800941.

(21) Doktycz, S. J.; Suslick, K. S. Interparticle Collisions Driven by Ultrasound. Science 1990, 247, 1067-1069.

(22) Prozorov, T.; Prozorov, R.; Suslick, K. S. High Velocity Interparticle Collisions Driven by Ultrasound. J. Am. Chem. Soc. 2004, 126, 13890-13891.

(23) Nicolosi, V.; Chhowalla, M.; Kanatzidis, M. G.; Strano, M. S.; Coleman, J. N. Liquid Exfoliation of Layered Materials. Science 2013, 340, 1226419.

(24) Chew, H. B.; Moon, M. W.; Lee, K. R.; Kim, K. S. Compressive Dynamic Scission of Carbon Nanotubes under Sonication: Fracture by Atomic Ejection. Proc. R. Soc. London, Ser. A 2011, 467, 12701289.

(25) Zhu, Y.; Li, L.; Zhang, C. G.; Casillas, G.; Sun, Z. Z.; Yan, Z.; Ruan, G. D.; Peng, Z. W.; Raji, A. R. O.; Kittrell, C.; Hauge, R. H.; Tour, J. M. A Seamless Three-Dimensional Carbon Nanotube Graphene Hybrid Material. Nat. Commun. 2012, 3, 1225.

(26) Bepete, G.; Anglaret, E.; Ortolani, L.; Morandi, V.; Huang, K.; Pénicaud, A.; Drummond, C. Surfactant-Free Single-Layer Graphene in Water. Nat. Chem. 2017, 9, 347-352.
(27) Yang, L.; Zhang, R.; Liu, B.; Wang, J.; Wang, S.; Han, M.-Y.; Zhang, Z. $\pi$-Conjugated Carbon Radicals at Graphene Oxide to Initiate Ultrastrong Chemiluminescence. Angew. Chem., Int. Ed. 2014, 53, 10109-10113.

(28) Suslick, K. S. The Chemical Effects of Ultrasound. Sci. Am. 1989, 260, 80-87.

(29) Li, Y.; Zhou, W.; Wang, H.; Xie, L.; Liang, Y.; Wei, F.; Idrobo, J.-C.; Pennycook, S. J.; Dai, H. An Oxygen Reduction Electrocatalyst Based on Carbon Nanotube-Graphene Complexes. Nat. Nanotechnol. 2012, 7, 394-400.

(30) Novaes, F. D.; Rurali, R.; Ordejon, P. Electronic Transport between Graphene Layers Covalently Connected by Carbon Nanotubes. ACS Nano 2010, 4, 7596-7602.

(31) Varshney, V.; Patnaik, S. S.; Roy, A. K.; Froudakis, G.; Farmer, B. L. Modeling of Thermal Transport in Pillared-Graphene Architectures. ACS Nano 2010, 4, 1153-1161.

(32) Tang, C.; Zhang, Q.; Zhao, M.-Q.; Huang, J.-Q.; Cheng, X.-B.; Tian, G.-L.; Peng, H.-J.; Wei, F. Nitrogen-Doped Aligned Carbon Nanotube/Graphene Sandwiches: Facile Catalytic Growth on Bifunctional Natural Catalysts and Their Applications as Scaffolds for HighRate Lithium-Sulfur Batteries. Adv. Mater. 2014, 26, 6100-6105.

(33) Yang, Y.; Kim, N. D.; Varshney, V.; Sihn, S.; Li, Y.; Roy, A. K.; Tour, J. M.; Lou, J. In Situ Mechanical Investigation of Carbon Nanotube-Graphene Junction in Three-Ddimensional Carbon Nanostructures. Nanoscale 2017, 9, 2916-2924.

(34) Fan, Z.; Yan, J.; Zhi, L.; Zhang, Q.; Wei, T.; Feng, J.; Zhang, M.; Qian, W.; Wei, F. A Three-Dimensional Carbon Nanotube/ Graphene Sandwich and Its Application As Electrode in Supercapacitors. Adv. Mater. 2010, 22, 3723-3728.

(35) Dimitrakakis, G. K.; Tylianakis, E.; Froudakis, G. E. Pillared Graphene: A New 3-D Network Nanostructure for Enhanced Hydrogen Storage. Nano Lett. 2008, 8, 3166-3170.

(36) Yan, Z.; Peng, Z.; Casillas, G.; Lin, J.; Xiang, C.; Zhou, H.; Yang, Y.; Ruan, G.; Raji, A.-R. O.; Samuel, E. L. G.; Hauge, R. H.; Yacaman, M. J.; Tour, J. M. Rebar Graphene. ACS Nano 2014, 8, 5061-5068.

(37) Li, J. F.; Huang, Y. F.; Ding, Y.; Yang, Z. L.; Li, S. B.; Zhou, X. S.; Fan, F. R.; Zhang, W.; Zhou, Z. Y.; Wu, D. Y.; Ren, B.; Wang, Z. L.; Tian, Z. Q. Shell-Isolated Nanoparticle-Enhanced Raman Spectroscopy. Nature 2010, 464, 392-395.

(38) Osipov, V. Y.; Baranov, A. V.; Ermakov, V. A.; Makarova, T. L.; Chungong, L. F.; Shames, A. I.; Takai, K.; Enoki, T.; Kaburagi, Y.; Endo, M.; Vul, A. Y. Raman Characterization and UV Optical Absorption Studies of Surface Plasmon Resonance in Multishell Nanographite. Diamond Relat. Mater. 2011, 20, 205-209.

(39) LopezRios, T.; Sandre, E.; Leclercq, S.; Sauvain, E. Polyacetylene in Diamond Films Evidenced by Surface Enhanced Raman Scattering. Phys. Rev. Lett. 1996, 76, 4935-4938.

(40) Ferrari, A. C.; Robertson, J. Origin of The $1150-\mathrm{cm}^{-1}$ Raman Mode in Nanocrystalline Diamond. Phys. Rev. B: Condens. Matter Mater. Phys. 2001, 63, 121405.

(41) Bogdanov, K.; Fedorov, A.; Osipov, V.; Enoki, T.; Takai, K.; Hayashi, T.; Ermakov, V.; Moshkalev, S.; Baranov, A. AnnealingInduced Structural Changes of Carbon Onions: High-Resolution Transmission Electron Microscopy and Raman Studies. Carbon 2014, $73,78-86$.

(42) Ferrari, A. C.; Basko, D. M. Raman Spectroscopy As A Versatile Tool for Studying The Properties of Graphene. Nat. Nanotechnol. 2013, 8, 235-246.

(43) Muthoosamy, K.; Manickam, S. State of the Art and Recent Advances in the Ultrasound-Assisted Synthesis, Exfoliation and Functionalization of Graphene Derivatives. Ultrason. Sonochem. 2017, 39, 478-493.

(44) Wei, Z.; Wang, D.; Kim, S.; Kim, S.-Y.; Hu, Y.; Yakes, M. K.; Laracuente, A. R.; Dai, Z.; Marder, S. R.; Berger, C.; King, W. P.; de Heer, W. A.; Sheehan, P. E.; Riedo, E. Nanoscale Tunable Reduction of Graphene Oxide for Graphene Electronics. Science 2010, 328, $1373-1376$ 
(45) Rooze, J.; Groote, R.; Jakobs, R. T. M.; Sijbesma, R. P.; van Iersel, M. M.; Rebrov, E. V.; Schouten, J. C.; Keurentjes, J. T. F. Mechanism of Ultrasound Scission of a Silver-Carbene Coordination Polymer. J. Phys. Chem. B 2011, 115, 11038-11043.

(46) Acik, M.; Lee, G.; Mattevi, C.; Pirkle, A.; Wallace, R. M.; Chhowalla, M.; Cho, K.; Chabal, Y. The Role of Oxygen during Thermal Reduction of Graphene Oxide Studied by Infrared Absorption Spectroscopy. J. Phys. Chem. C 2011, 115, 19761-19781.

(47) Fabre, N.; Rustan, I.; de Hoffmann, E.; Quetin-Leclercq, J. Determination of Flavone, Flavonol, and Flavanone Aglycones by Negative Ion Liquid Chromatography Electrospray Ion Trap Mass Spectrometry. J. Am. Soc. Mass Spectrom. 2001, 12, 707-715.

(48) An, S. J.; Zhu, Y.; Lee, S. H.; Stoller, M. D.; Emilsson, T.; Park, S.; Velamakanni, A.; An, J.; Ruoff, R. S. Thin Film Fabrication and Simultaneous Anodic Reduction of Deposited Graphene Oxide Platelets by Electrophoretic Deposition. J. Phys. Chem. Lett. 2010, 1, $1259-1263$.

(49) Wan, J.; Jiang, J.-W.; Park, H. S. Negative Poisson's Ratio in Graphene Oxide. Nanoscale 2017, 9, 4007-4012.

(50) Jiang, J. W.; Chang, T. C.; Guo, X. M. Tunable Negative Poisson's Ratio in Hydrogenated Graphene. Nanoscale 2016, 8, $15948-15953$.

(51) Frank, O.; Tsoukleri, G.; Riaz, I.; Papagelis, K.; Parthenios, J.; Ferrari, A. C.; Geim, A. K.; Novoselov, K. S.; Galiotis, C. Development of A Universal Stress Sensor for Graphene and Carbon Fibres. Nat. Commun. 2011, 2, 255.

(52) Huang, M.; Yan, H.; Chen, C.; Song, D.; Heinz, T. F.; Hone, J. Phonon Softening and Crystallographic Orientation of Strained Graphene Studied by Raman Spectroscopy. Proc. Natl. Acad. Sci. U. S. A. 2009, 106, 7304-7308.

(53) Lee, J. E.; Ahn, G.; Shim, J.; Lee, Y. S.; Ryu, S. Optical Separation of Mechanical Strain from Charge Doping in Graphene. Nat. Commun. 2012, 3, 1024.

(54) Pisana, S.; Lazzeri, M.; Casiraghi, C.; Novoselov, K. S.; Geim, A. K.; Ferrari, A. C.; Mauri, F. Breakdown of The Adiabatic BornOppenheimer Approximation in Graphene. Nat. Mater. 2007, 6, 198201.

(55) Gao, W.; Alemany, L. B.; Ci, L.; Ajayan, P. M. New Insights into The Structure and Reduction of Graphite Oxide. Nat. Chem. 2009, 1, 403-408.

(56) Lin, C. Y.; Zhao, Z. H.; Niu, J. B.; Xia, Z. H. Synthesis, Properties and Applications of 3D Carbon Nanotube-Graphene Junctions. J. Phys. D: Appl. Phys. 2016, 49, 443001.

(57) Xu, Z.-C.; Zhong, W.-R. Probability of Self-Healing in Damaged Graphene Bombarded by Fullerene. Appl. Phys. Lett. 2014, 104, 261907.

(58) Yang, X.; Wang, L.; Huang, Y.; Han, Z.; To, A. C. Carbon Nanotube-Fullerene Hybrid Nanostructures by C60 Bombardment: Formation and Mechanical Behavior. Phys. Chem. Chem. Phys. 2014, $16,21615-21619$.

(59) Kucukkal, M. U.; Stuart, S. J. Simulation of Carbon Nanotube Welding through Ar Bombardment. J. Mol. Model. 2017, 23, 148. 